\section{Pet ownership and cognitive decline in older people}

\author{
Nicola Veronese, ${ }^{1,2}$ Lee Smith, ${ }^{3}$ \\ Vania Noventa, ${ }^{1}$ \\ Guillermo F. López-Sánchez, ${ }^{4}$ \\ Jacopo Demurtas, ${ }^{5}$ \\ Christopher F. Sharpley, ${ }^{6}$ Vicki Bitsika, ${ }^{7}$ \\ Sarah E. Jackson ${ }^{8}$ \\ ${ }^{1}$ Primary Care Department, Geriatric \\ Unit, Azienda ULSS (Unità Locale Socio \\ Sanitaria) 3 Serenissima, Dolo-Mirano \\ District, Italy; ${ }^{2}$ National Research \\ Council, Neuroscience Institute, Aging \\ Branch, Padua, Italy; ${ }^{3}$ Cambridge \\ Centre for Sport and Exercise Sciences, \\ Anglia Ruskin University, Compass \\ House, Cambridge, UK; ${ }^{4}$ Faculty of \\ Sport Sciences, University of Murcia, \\ Spain; ${ }^{5}$ Primary Care Department, \\ Azienda USL Toscana Sud Est, Grosseto, \\ Italy; ${ }^{6}$ Brain-Behaviour Research \\ Group, University of New England, New \\ South Wales, Australia; ${ }^{7}$ Centre for \\ Autism Spectrum Disorder, Bond \\ University, Queensland, Australia; \\ ${ }^{8}$ Department of Behavioural Science and \\ Health, UCL, London, UK
}

\section{Abstract}

Animals can have a positive influence on human health. However, it is not yet known whether pet ownership can prevent cognitive decline. Therefore, we aimed to investigate cross-sectional and prospective associations between pet ownership and cognitive function in a large, representative sample of older adults.

Data were from the English Longitudinal Study of Ageing (ELSA) using data collected in wave 5 and six years later in wave 8 . Pet ownership was categorized as no pet, dog, cat or other pet. Cognitive function was assessed using tests of verbal fluency (assessed by asking how many different animals the participants could name in 60 seconds) and memory (sum of immediate and delayed verbal recall). Multiple linear regression, adjusted for potential confounders, was used to test the associations between pet ownership and cognitive function.

A total of 8291 people (mean age: 66.72 years) were included. In cross-sectional analyses, dog owners had better verbal fluency than individuals with no pet, but there was no significant difference between cat or other pet owners and those with no pet. In prospective analyses, dog owners had a significantly larger decline in recall than those with no pet, whilst cat owners had a significantly smaller decline in verbal fluency.

These results provide some evidence to suggest that pet ownership may have positive effects on cognition in later life. However, benefits of pet ownership were not unilaterally observed across different types of pet and measures of cognitive function suggesting that further research is required.

\section{Introduction}

It has widely been reported that animals can have a positive influence on human health. ${ }^{1}$ In this sense, animals are involved in a variety of health care settings, and are often introduced to individuals struggling with a malady. ${ }^{1}$ For example, some physicians use animals as an adjunct to physical therapy by having a patient walk a dog, pet or brush a cat, or play fetch with a dog. ${ }^{2}$ Interactions with an animal can serve to realize specific physical therapy goals, and the animal's unique ability to be attentive to the client may serve to increase interest in activities and improve mental health functioning. ${ }^{2}$ Animals have been largely used in mental health settings to achieve a variety of benefits: for example, a child may be encouraged to gently pet and talk to an animal to teach appropriate touch, reduce anxiety, increase a sense of connection to a living being, reduce loneliness, and develop a variety of skills. ${ }^{2}$

Animals are also used to support older people affected by dementia. Several small studies have shown that the presence of a dog can reduce aggression and agitation and promote social behaviour in people affected by dementia, suggesting that pets could play an important role in decreasing behavioural and psychological symptoms of dementia (BPSD). ${ }^{3}$ However, data regarding the impact of pet ownership on cognition are limited to one small study. One study found that in 100 nursing home residents, biweekly dog visits were not able to improve MMSE scores over 6 weeks of intervention, ${ }^{4}$ but it is likely that this period was too short to detect any significant change in cognitive performance. Understanding whether pet ownership could help protect against age-related cognitive decline in older people is of importance, since dementia prevalence is increasing in industrialized countries ${ }^{5}$ and owning a pet is a relative inexpensive intervention.

Given this background, we aimed to investigate the possible cross-sectional and
Correspondence: Nicola Veronese, National Research Council, Neuroscience Institute, Aging Branch, via Giustiniani 2, 35128 Padua, Italy. Tel.: +39.0498211746. E-mail: ilmannato@gmail.com

Key words: Pet ownership; cat; dog; dementia; cognition; ELSA.

Contributions: All authors listed (NV, LS, VN, GFLS, JD, CFS, VB, SEJ) have made a substantial, direct and intellectual contribution to the work, and approved it for publication.

Conflict of interests: the authors declare no potential conflict of interests.

Received for publication: 17 September 2019. Accepted for publication: 1 October 2019.

This work is licensed under a Creative Commons Attribution-NonCommercial 4.0 International License (CC BY-NC 4.0).

${ }^{\circ}$ Copyright: the Author(s), 2019

Licensee PAGEPress, Italy

Geriatric Care 2019; 5:8568

doi: $10.4081 /$ gc. 2019.8568

prospective associations between pet ownership and cognitive decline in a large, representative sample of older adults living in England. Specifically, we analysed associations between cat, dog or other animals' ownership and the rate of decline in memory and verbal fluency.

\section{Materials and Methods \\ Study population}

Data were from the English Longitudinal Study of Ageing (ELSA), an ongoing longitudinal panel study of men and women aged $\geq 50$ years recruited from households in England. ELSA's sample and methods have been described in detail elsewhere, ${ }^{6}$ but briefly, the study began in 2002 and data have been collected every two years since via computer-assisted personal interview and self-completion questionnaires. For the purpose of the present analyses, baseline data were drawn from Wave 5 (2010/11; the first wave in which participants were asked about pet ownership) and follow-up data were from Wave 8 (2016/17; the latest wave of data available). Of the 10,317 participants interviewed in Wave 5, $8291(80.4 \%)$ had complete data on pet ownership, all covariates and at least one measure of cognitive function and formed our analytic sample. Follow-up data were available for $5725(69.1 \%$ of the baseline sample). 


\section{Measures}

\section{Exposure: pet ownership}

Pet ownership was assessed with two questions: i) Do you keep any household pets inside your house/flat?; and if yes ii) What pets do you keep inside your house/flat? (dog/cat/bird/other furry pets/other). Because the latter three types of pet were infrequently reported, we analysed data using a four-level variable: no pet, dog, cat or other pet.

\section{Outcome: change in cognitive function}

Cognitive function is evaluated in ELSA using a variety of tests, but these vary across data collection waves. For our research, we focused on memory and verbal fluency as indicators of cognitive function because these tests were included in both the Wave 5 and Wave 8 assessments. Memory was calculated as the sum of immediate and delayed verbal memory. Each participant was presented with a list of 10 nouns on a computer, one every two seconds. Participants were asked to recall as many words as possible immediately and again after a short delay during which they carried out other cognitive tests. Verbal fluency was assessed by asking how many different animals the participants could name in one minute.

For each outcome, in order to calculate the degree of cognitive change between Wave 5 and Wave 8 we carried out a linear regression analysis using the values of each test at Wave 5 as independent variables and scores of cognitive tests at Wave 8 as dependent variables, and used the standardised residual as a measure of cognitive change, as has been done in previous work in this area. ${ }^{7}$

\section{Covariates}

Demographic information collected included age, sex, ethnicity, highest level of education, marital status and socioeconomic status (SES). Ethnicity was categorised as white vs. non-white. Education was categorised as no qualifications, up to O level, A level and foreign, higher below degree, or degree or higher. Marital status was categorised as married/living as married, single/never married, divorced/separated, or widowed. SES was indexed using household non-pension wealth (in quintiles calculated in the entire ELSA population), which has been identified as a sensitive indicator in this population. ${ }^{8}$ Physical function was assessed based on participants' responses to questions on perceived difficulty performing basic activities of daily living (e.g. difficulty dressing, including putting on shoes and socks) and was categorised as any vs. no difficulty. ${ }^{9}$ Depressive symptoms were assessed using the eight-item Centre for Epidemiological Studies Depression Scale, a scale validated for use in older adults. ${ }^{10}$ Participants reported their current smoking status (smoker vs. nonsmoker) and level of physical activity (categorised as: inactive [no moderate/vigorous activity on a weekly basis], moderate activity at least once a week, and vigorous activity at least once a week. ${ }^{11}$ Finally, participants reported the presence or absence of limiting long-standing illness, defined as any longstanding illness, disability, or infirmity that limits activities in any way.

\section{Statistical analysis}

Analyses were performed using IBM SPSS Statistics 25. Data were weighted to correct for sampling probabilities and for differential non-response and to calibrate back to the 2011 National Census population distributions for age and sex. For cross-sectional analyses, the weights accounted for the differential probability of being included in Wave 5 of ELSA. For prospective analyses, we applied a longitudinal weight that accounted for nonresponse at Wave 8 based on the sample who participated in Wave 4.

Associations between pet ownership and covariates (measured at baseline) were analysed using one-way independent analysis of variance (continuous variables) and chi-square tests (categorical variables). We used multiple linear regression to test the cross-sectional association between pet ownership and cognitive function at baseline, and the prospective association between pet ownership at baseline and change in cognitive function over six-year follow-up. We ran two sets of models: the first were minimally adjusted for age and sex, the second were adjusted for age, sex, ethnicity, education, marital status, wealth, physical function, depressive symptoms, smoking status, physical activity and limiting long-standing illness. All prospective analyses were additionally adjusted for baseline score on the outcome of interest. The reference category was no pet. Results are reported as unstandardized B values with $95 \%$ confidence intervals (CIs), which can be interpreted as the adjusted mean difference between each pet ownership group and non-pet owners.

\section{Results}

Our sample comprised 8291 men and women aged between 52 and 89 years (mean age 66.72 , SD 9.18 years). The majority $(66.2 \%, n=5492)$ reported having no pet, $18.2 \% \quad(n=1505)$ owned a dog, $12.5 \%$ $(n=1033)$ owned a cat, and 3.1\% $(n=261)$ owned another type of pet. Sample character- istics in relation to pet ownership are summarised in Table 1. Pet owners were on average significantly younger than those with no pet, and a higher proportion were white and married. Cat owners tended to be more highly educated and wealthy than the other groups, and other pet owners were the least wealthy. Dog owners reported more depressive symptoms on average than the other groups and were most likely to smoke and have a limiting long-standing illness. Individuals with no pet were most likely to be inactive. There was no significant association between pet ownership and sex or physical function.

Cross-sectional and prospective associations between pet ownership and cognitive function are shown in Table 2. Cross-sectionally, there was no significant association between pet ownership and recall in either the minimally-adjusted or fully-adjusted models. There was no significant association between pet ownership and fluency in the minimally-adjusted model, but in the fullyadjusted model dog owners had significantly better verbal fluency than those with no pet. Prospectively, dog owners had a significantly larger decline in recall than those with no pet in both minimally-adjusted and fully-adjusted models. Other pet owners also had a larger decline recall than those with no pet in the minimally-adjusted model but the difference was not statistically significant in the fullyadjusted model. Cat owners had a significantly smaller decline in verbal fluency than those with no pets in both minimally-adjusted and fully-adjusted models. Dog owners also had a marginally smaller decline in fluency in the fully-adjusted model, but the difference was not statistically significant.

\section{Discussion and Conclusions}

In this large study, we found some evidence to suggest that pet ownership is associated with a better cognitive profile, but benefits were not consistently observed across categories of pet and different cognitive tests. At baseline, there was little difference in memory performance between older people with and without pets, but dog owners had significantly better verbal fluency than those with no pet. In prospective analyses, we observed a smaller decline in verbal fluency among cat owners, but a greater decline in recall among dog owners.

Accumulating evidence describes potential benefits of animals for people with dementia and cognitive impairment. In recent years, animals have been introduced into the care of people affected by dementia $^{12}$ and have been shown to serve as an 
extended arm for social interaction between humans. ${ }^{13}$ In people affected by dementia, the presence of an animal can also help to facilitate contact between caregiver and patient. ${ }^{12}$ Animals are used in dementia care for increasing social behaviour and quality of life, ${ }^{14}$ reducing aggression and anxiety, ${ }^{15}$ and for increasing physical activity among people with dementia. ${ }^{12}$ Spending time with animals may evoke memories and offer a sense of physical closeness during the time the person spends stroking the animal. ${ }^{12}$
However, just one previous study had examined the influence of exposure to domestic animals on cognitive function, and found no significant association, ${ }^{4}$ but the study period was short (6 weeks) and the sample comprised nursing home residents

Table 1. Sample characteristics in relation to pet ownership.

\begin{tabular}{|c|c|c|c|c|c|}
\hline & No $\operatorname{pet}(\mathrm{n}=5492) *$ & $\operatorname{Dog}(n=1505)$ & Cat(n=1033) & Other $(n=261)$ & $\mathbf{P}$ \\
\hline Age (mean $[\mathrm{SD}]$ years) & $68.35(9.13)$ & $64.36(7.72)$ & $64.49(8.21)$ & $63.60(8.29)$ & $<0.001$ \\
\hline Female sex $(\%)$ & 52.6 & 53.6 & 53.5 & 50.4 & 0.714 \\
\hline Non-white ethnicity (\%) & 4.8 & 1.2 & 2.6 & 2.1 & $<0.001$ \\
\hline $\begin{array}{l}\text { Highest level of education (\%) } \\
\text { No qualifications } \\
\text { O level } \\
\text { A level or foreign } \\
\text { Higher below degree } \\
\text { Degree or higher } \\
\end{array}$ & $\begin{array}{l}29.9 \\
22.5 \\
15.4 \\
15.0 \\
17.2 \\
\end{array}$ & $\begin{array}{l}28.1 \\
25.7 \\
15.7 \\
15.4 \\
15.1\end{array}$ & $\begin{array}{l}21.2 \\
26.4 \\
16.2 \\
16.1 \\
20.0\end{array}$ & $\begin{array}{l}29.1 \\
27.0 \\
16.7 \\
11.3 \\
16.0\end{array}$ & $\begin{array}{c}<0.001 \\
- \\
- \\
- \\
-\end{array}$ \\
\hline $\begin{array}{l}\text { Marital status (\%) } \\
\text { Married/living as married } \\
\text { Single/never married } \\
\text { Divorced } \\
\text { Widowed }\end{array}$ & $\begin{array}{c}64.7 \\
6.6 \\
11.8 \\
16.9\end{array}$ & $\begin{array}{c}73.0 \\
4.3 \\
12.1 \\
10.6\end{array}$ & $\begin{array}{c}67.7 \\
6.6 \\
14.8 \\
10.9\end{array}$ & $\begin{array}{c}71.4 \\
6.4 \\
10.6 \\
11.7\end{array}$ & $\begin{array}{c}<0.001 \\
- \\
- \\
-\end{array}$ \\
\hline $\begin{array}{l}\text { Wealth quintile (\%) } \\
\qquad \begin{array}{l}1 \text { (poorest) } \\
2 \\
3 \\
4 \\
5 \text { (richest) }\end{array}\end{array}$ & $\begin{array}{l}18.1 \\
20.1 \\
21.3 \\
20.2 \\
20.2\end{array}$ & $\begin{array}{l}23.6 \\
21.2 \\
17.0 \\
18.6 \\
19.6\end{array}$ & $\begin{array}{l}16.7 \\
18.5 \\
19.7 \\
21.9 \\
23.1\end{array}$ & $\begin{array}{l}29.4 \\
16.7 \\
19.9 \\
19.9 \\
14.2\end{array}$ & $\begin{array}{c}<0.001 \\
- \\
- \\
- \\
-\end{array}$ \\
\hline Physical function limitation (\%) & 18.6 & 19.4 & 15.7 & 17.0 & 0.086 \\
\hline Depressive symptoms (0-8) (mean [SD] years) & $1.48(1.91)$ & $1.71(2.16)$ & $1.51(2.00)$ & $1.53(2.11)$ & 0.001 \\
\hline Smoker (\%) & 12.0 & 20.5 & 16.2 & 16.7 & $<0.001$ \\
\hline $\begin{array}{l}\text { Physical activity (\%) } \\
\text { Inactive } \\
\text { Moderately active at least once a week } \\
\text { Vigorously active at least once a week }\end{array}$ & $\begin{array}{l}26.4 \\
46.3 \\
27.3\end{array}$ & $\begin{array}{l}21.7 \\
50.6 \\
27.7\end{array}$ & $\begin{array}{l}20.2 \\
46.6 \\
33.2\end{array}$ & $\begin{array}{l}23.4 \\
46.5 \\
30.1\end{array}$ & $\begin{array}{c}<0.001 \\
- \\
-\end{array}$ \\
\hline Limiting long-standing illness (\%) & 34.7 & 37.6 & 31.1 & 34.8 & 0.008 \\
\hline
\end{tabular}

All figures are weighted to match the older English population. *Unweighted sample sizes. SD, standard deviation.

Table 2. Cross-sectional and prospective associations between pet ownership and cognitive function.

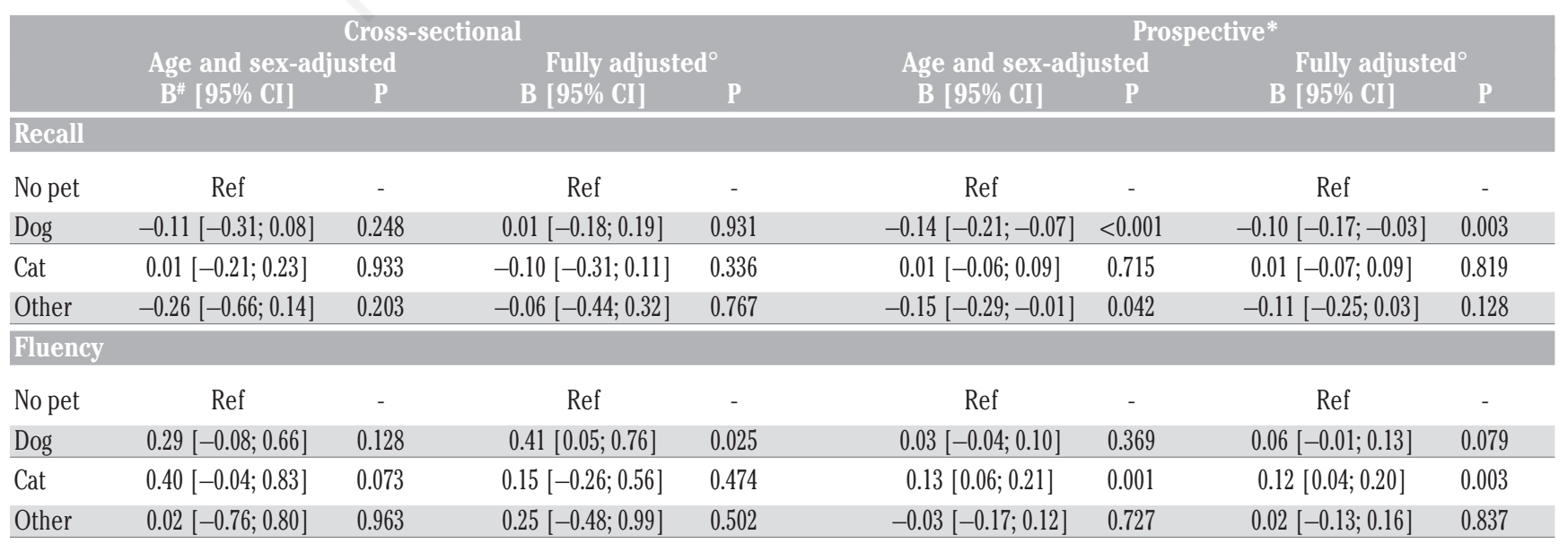

All figures are weighted to match the older English population. *Prospective models test change in cognitive function between baseline and follow-up and are additionally adjusted for baseline score. ${ }^{\circ}$ Fully adjusted models are adjusted for age, sex, ethnicity, education, marital status, wealth, physical function, depressive symptoms, smoking status, physical activity and limiting long-standing illness. "In cross-sectional results, a negative $B$ value indicates poorer cognitive function and a positive B value indicates better cognitive function, relative to the no pet group. In prospective results, a negative B value indicates a greater decline in cognitive function and a positive B value indicates a smaller decline in cognitive function, relative to the no pet group. Cl, confidence interval. 
visited by a dog, rather than free-living adults who own their own pet. Our results build upon this existing literature, demonstrating some evidence of benefits of pet ownership for cognitive performance over a follow-up period of six years.

The inconsistencies in our findings may, to some extent, be explained by differences in the characteristics of the analysed groups. First, cat owners tended to be more highly educated and wealthy than the other groups. Education is an important factor in predicting cognitive impairment and dementia in older people. ${ }^{16}$ For example, the mini-mental state examination (MMSE), a common tool for the diagnosis of dementia, is stratified for the years of education. ${ }^{17}$ Therefore, it is possible that the association we found for cat owners during the follow-up period reflects a larger cognitive reserve, due to higher educational level and a higher socio-economic status per se. The association between cat ownership and decline in verbal fluency was attenuated somewhat when these variables were included in the model, indicating that they likely play a role, although a significant different persisted after adjustment, indicating that there are other implicated factors that we did not adjust for. An alternative explanation may be that younger older adults are more likely to own cats and thus have a greater cognitive reserve at baseline. ${ }^{18} \mathrm{Cat}$ owners are more likely to live in semi urban/rural settings and access to green space has been shown to be beneficial for cognitive ageing. ${ }^{19}$ Finally, some large panel surveys have shown that cat owners enjoy leisurely activities such as reading and writing that may protect against the aging decline in cognition. ${ }^{20}$ We cannot rule out that those who own cats may engage in certain activities with their pet that may protect against cognitive decline, however no literature exists to support this hypothesis and further research is needed.

Associations between dog ownership and cognitive performance were mixed. Cross-sectionally, dog owners scored better on a test of verbal fluency than individuals with no pet, but prospectively, they had a greater decline in memory. The reasons for these seemingly contradictory results are not clear. It is possible that pet ownership has benefits for some aspects of cognition (e.g. verbal fluency, as was also observed in cat owners in prospective analyses), but not others (e.g. memory). Alternatively, the greater decline in recall in dog owners could be a spurious finding. Further research is required to replicate these results. If it is a valid finding, some baseline characteristics that were less favourable among dog owners may be implicated. At baseline, dog owners reported a higher number of depressive symptoms than those with no pet and were more likely to have a limiting long-standing illness; factors known to be associated with cognitive decline. For this specific group, we could therefore argue a reverse causality, i.e. people who are more depressed and more disabled have sought out a dog prior to our baseline evaluation. Existing literature suggests that dogs can have prophylactic and therapeutic value for people having depression or anxiety. ${ }^{21}$ Thus, it is possible that these people have acquired a dog for companionship and to improve symptoms of depression. Moreover, individuals who have limiting long-standing illness may acquire a dog for home safety and security (e.g. if they have a disability). While adjusting for these factors attenuated the association, it remained significant after adjustment. There is a need for further research to explore other potential confounders and/or mediators of this relationship.

To the best of our knowledge, this is the first study to explore the potential association between pet ownership and cognitive decline in older people. Strengths of the study include the large, representative sample and data on a wide range of potential confounders. However, findings from the present study must be interpreted considering its limitations. First, many measures (e.g. those regarding physical activity and comorbidities) were self-reported, which might have introduced reporting or recall bias. Second, the sample was almost exclusively white, so findings may not generalise to other ethnic groups. Finally, we can also consider a lack of power (type II error) for some groups included in this study and owning a pet, such as those having animals other than cats and dogs. Therefore, other studies are needed in this sense.

In conclusion, the present study provides some evidence that pet ownership can have positive effects on cognition in older people. However, benefits of pet ownership were not unilaterally observed across different types of pet and measures of cognitive function. Further research is required to establish the exact nature of the relationship between pet ownership and cognitive function and provide insight into the causal pathways underpinning differences across pet subgroups and dimensions of cognition.

\section{References}

1. Nimer J, Lundahl B. Animal-assisted therapy: A meta-analysis. Anthrozoös 2007;20:225-38.
2. Chandler CK. Animal assisted therapy in counseling. London: Routledge; 2012.

3. Filan SL, Llewellyn-Jones RH. Animalassisted therapy for dementia: a review of the literature. Int Psychogeriatr 2006;18:597-611.

4. Thodberg K, Sørensen LU, Christensen JW, et al. Therapeutic effects of dog visits in nursing homes for the elderly. Psychogeriatrics 2016;16:289-97.

5. Alzheimer's A. 2015 Alzheimer's disease facts and figures. Alzheim Dementia 2015;11:332-84.

6. Steptoe A, Breeze E, Banks J, et al. Cohort profile: the English longitudinal study of ageing. Int $\mathrm{J}$ Epidemiol 2012;42:1640-8.

7. Veronese N, Koyanagi A, Solmi M, et al. Pain is not associated with cognitive decline in older adults: A four-year longitudinal study. Maturitas 2018; 115:92-6.

8. Banks J, Karlsen S, Oldfield Z. Socioeconomic position. Institute for Fiscal Studies; 2003.

9. Katz S, Downs TD, Cash HR, et al. Progress in development of the index of ADL. The Gerontologist 1970;10:20-30.

10. Steffick D, Group HW. Documentation of affective functioning measures in the Health and Retirement Study. HRS/AHEAD Documentation Report DR-005 [online report]; 2000, 2009.

11. Hamer M, Molloy GJ, de Oliveira C, et al. Leisure time physical activity, risk of depressive symptoms, and inflammatory mediators: the English Longitudinal Study of Ageing. Psychoneuroendocrinology 2009;34:1050-5.

12. Swall A, Ebbeskog B, Lundh Hagelin C, et al. Stepping out of the shadows of Alzheimer's disease: a phenomenological hermeneutic study of older people with Alzheimer's disease caring for a therapy dog. Int J Qual Stud Health Well-being 2017;12:1347013.

13. Fine AH. Handbook on animal-assisted therapy: Foundations and guidelines for animal-assisted interventions. Massachusetts: Academic Press; 2015.

14. Bernabei V, De Ronchi D, La Ferla T, et al. Animal-assisted interventions for elderly patients affected by dementia or psychiatric disorders: a review. J Psychiatr Res 2013;47:762-73.

15. Kanamori M, Suzuki M, Yamamoto K, et al. A day care program and evaluation of animal-assisted therapy (AAT) for the elderly with senile dementia. Am J Alzheimers Dis Other Demen 2001;16: 234-9.

16 Sharp ES, Gatz M. The relationship between education and dementia an updated systematic review. Alzheimer 
Dis Assoc Disord 2011;25:289-304. 17. Tombaugh TN, McIntyre NJ. The minimental state examination: a comprehensive review. J Am Geriatr Soc 1992; 40:922-35.

18. Murray JK, Browne WJ, Roberts MA, et al. Number and ownership profiles of cats and dogs in the UK. Vet Rec 2010; 166:163-8.

19. Cherrie MP, Shortt NK, Mitchell RJ, et al. Green space and cognitive ageing: A retrospective life course analysis in the Lothian Birth Cohort 1936. Soc Sci Med 2018;196:56-65.
20. Nutrition WCfP. 2018 Available from: https://www.mars.com/global/scienceand-innovation/science/petcare Accessed: 14/12/18 2018.

21. Wells DL. Domestic dogs and human health: An overview. $\mathrm{Br} \mathrm{J}$ Health Psychol 2007;12:145-56. 